\title{
Cost-effectiveness of aortic valve replacement in the elderly: An introductory study
}

\author{
YingXing Wu, MD, Ruyun Jin, MD, Guangqiang Gao, MD, Gary L. Grunkemeier, PhD, and Albert Starr, MD
}

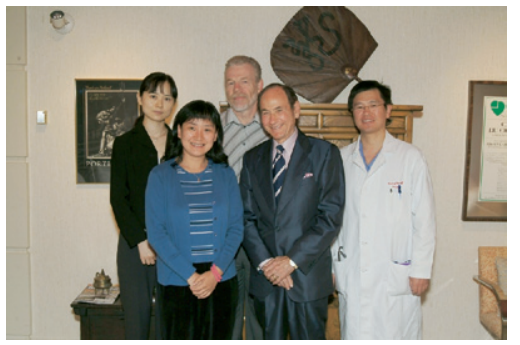

Drs Wu, Jin, Grunkemeier, Starr, and Gao (left to right)

See related articles on pages 601 and 603 .

\footnotetext{
From the Providence Heart and Vascular Institute, Providence Health System, Portland, Ore.

Received for publication April 17, 2006; revisions received Oct 11, 2006; accepted for publication Oct 24, 2006.

Address for reprints: YingXing Wu, Providence Health System, 9205 SW Barnes, Suite 33, Portland, OR 97225 (E-mail: yingxing.wu@providence.org).

J Thorac Cardiovasc Surg 2007;133:608-13 $0022-5223 / \$ 32.00$

Copyright $\odot 2007$ by The American Association for Thoracic Surgery

doi:10.1016/j.jtcvs.2006.10.044
}

Objective: With increased life expectancy and improved technology, valve replacement is being offered to increasing numbers of elderly patients with satisfactory clinical results. By using standard econometric techniques, we estimated the relative cost-effectiveness of aortic valve replacement by drawing on a large prospective database at our institution. By using aortic valve replacement as an example, this introductory report paves the way to more definitive studies of these issues in the future.

Methods: From 1961 to 2003, 4617 adult patients underwent aortic valve replacement at our service. These patients were provided with a prospective lifetime follow-up. As of 2005, these patients had accumulated 31,671 patient-years of follow-up (maximum 41 years) and had returned 22,396 yearly questionnaires. A statistical model was used to estimate the future life years of patients who are currently alive. In the absence of direct estimates of utility, quality-adjusted life years were estimated from New York Heart Association class. The cost-effectiveness ratio was calculated by the patient's age at surgery.

Results: The overall cost-effectiveness ratio was approximately $\$ 13,528$ per qualityadjusted life year gained. The cost-effectiveness ratio increased according to age at surgery, up to $\$ 19,826$ per quality-adjusted life year for octogenarians and $\$ 27,182$ per quality-adjusted life year for nonagenarians.

Conclusions: Given the limited scope of this introductory study, aortic valve replacement is cost-effective for all age groups and is very cost-effective for all but the most elderly according to standard econometric rules of thumb.

$\mathrm{T}$ The first successful heart valve replacement was performed in $1960 .{ }^{1}$ Currently, approximately 75,000 heart valve replacements are performed per year in the United States. Heart valve replacement has long been proven to be clinically effective in extending life expectancy and improving quality of life. It is increasingly performed in older patients, including those aged more than 80 years or even more than 90 years. At our institution, the mean age of patients undergoing aortic valve replacement (AVR) increased from 47 years to 69 years during the past 4 decades (Figure 1). The clinical effectiveness of this technique has been well documented, but the cost-effectiveness, to our knowledge, has not. Because this introductory study is the first venture into this new area, we used standard econometric techniques to estimate the relative cost-effectiveness of AVR, especially for the elderly.

\section{Materials and Methods}

From 1961 to 2003, 4617 patients aged 20 years or more underwent AVR at 3 hospitals in Portland, Oregon. Thirty-four percent of the patients had concomitant coronary artery bypass grafting, and none of the patients had other concomitant valve-replacement surgeries. Fortyseven percent of patients underwent AVR for aortic stenosis, $14 \%$ for aortic insufficiency, and 


\section{Abbreviations and Acronyms \\ AVR = aortic valve replacement \\ CER $=$ cost-effectiveness ratio \\ NYHA $=$ New York Heart Association \\ $\mathrm{QALY}=$ quality-adjusted life year}

$39 \%$ for both aortic stenosis and insufficiency. The mean age at surgery was 65 years (range 20-94 years), 502 patients (10.9\%) were octogenarians, and 23 patients $(0.5 \%)$ were nonagenarians (Figure 2).

Beginning with the first patient who underwent valve replacement, a prospective lifetime follow-up service was implemented. All patients undergoing valve replacement are followed up at annual intervals using a combination of mailed questionnaires and telephone interviews. The yearly surveys include questions regarding complications and medications. They also include questions regarding angina, shortness of breath, and daily activity for ascertainment of the New York Heart Association functional class (NYHA). As of the end of 2005, these patients had accumulated 31,671 follow-up years with a maximum of 41 years and had returned 22,396 yearly questionnaires.

Among the 4617 patients who underwent AVR, 2382 were documented as dead, and the other 2235 were still being followed at the time of the study (statistically, these patients are termed "censored"). To estimate the complete lifetimes of patients who have not yet died, a time of death was simulated using a Gompertz parametric regression model. Gompertz regression has been widely used to model survival time in survival analysis, especially for elderly persons. ${ }^{2}$ The model was developed using data from all patients who underwent heart valve replacement, and the predictors included age, gender, valve implant position, and concomitant coronary artery bypass grafting.

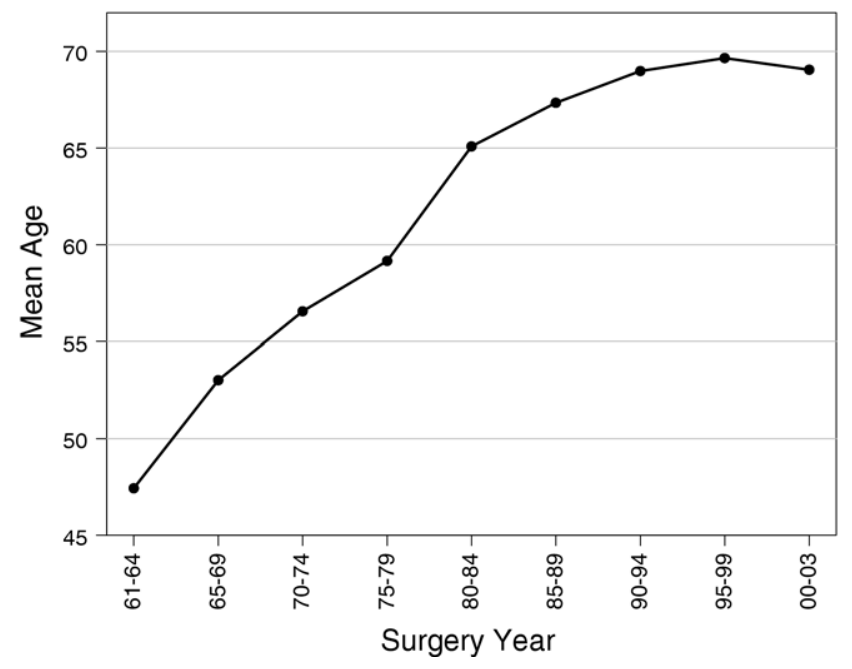

Figure 1. Mean age of patients who underwent AVR by surgery year. AVR, aortic valve replacement.

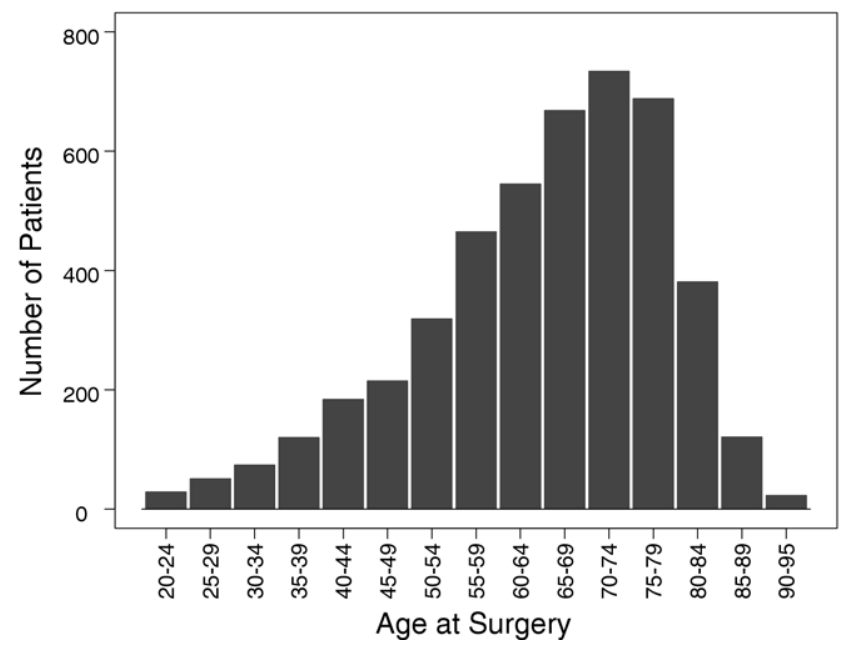

Figure 2. Number of patients who underwent AVR by age at surgery. AVR, aortic valve replacement.

\section{Cost-effectiveness Ratio: Net Cost of Additional Quality-adjusted Life Years}

The cost-effectiveness of AVR was assessed by comparing the surgical results with the natural history of patients with unoperated heart disease who would have been surgical candidates. The cost-effectiveness ratio (CER) was calculated for each age at surgery as CER $=(\mathrm{A}-\mathrm{B}) /(\mathrm{C}-\mathrm{D})$ (defined below), and the results were presented by grouping 5-year age intervals.

A. The lifetime costs of heart valve replacement

B. The lifetime costs of maintaining patients with unoperated heart valve disease

C. The quality-adjusted life years (QALYs) experienced after heart valve replacement

D. The QALYs experienced by patients with unoperated heart valve disease

A. The lifetime costs of AVR consist of 3 major components: (1) the cost of the original surgery; (2) the costs of ongoing maintenance (physician visits, echocardiograms, and anticoagulation therapy); and (3) the costs of treating valve-related complications (thromboembolism, bleeding, endocarditis, perivalvular leak, valve thrombosis, valve explant, and reimplantation). The cost of AVR surgery was approximately $\$ 60,000$ on average in 2005 at our service, and the cost of reoperation was set to be the same as the original surgery. Other numbers were extracted from the literature ${ }^{3}$ and converted to 2005 dollars using yearly inflation factors obtained from the consumer price index, which is complied by the Bureau of Labor Statistics (http://inflationdata.com/Inflation/Inflation_Rate/HistoricalInflation.aspx). For pa- 
tients who are currently alive, future life years were simulated using the Gompertz statistical model. To project the cost during these simulated future life years, physician visits and echocardiograms were assumed to occur annually; valve-related events rates were assumed to occur at the same constant rates as during the observed patient-years.

B. The costs of maintaining patients with unoperated heart valve disease was set at zero, because the numbers were difficult to obtain or estimate. Note that this has the effect of overestimating the CER.

C. The QALYs were estimated by mapping quality-oflife scores to NYHA functional class. Life years spent in NYHA classes I, II, III, and IV were estimated to be equivalent to $0.85,0.71,0.57$, and 0.43 QALYs, respectively. ${ }^{4}$ NYHA classes during follow-up were assessed by follow-up questionnaires regarding angina, shortness of breath, and daily activity. Follow-up questionnaires were usually obtained annually, but when not available for a certain year, the information was generated by linear interpolation from available years. For future simulated life years, NYHA classes were linearly interpolated between the latest available NYHA classes and class IV (assumed for the end of life).

D. The life years of patients with unoperated heart valve disease were estimated using a systematic review of the English language literature concerning the natural history of heart valve disease in the aortic position. The literature search was performed using Medline, the American College of Cardiology/America Heart Association guidelines for the management of patients with valvular heart disease, ${ }^{5}$ and an article by Kloster and Morris ${ }^{6}$ containing key references of the natural history of valvular heart disease. The study inclusion criteria were as follows: (1) The Kaplan-Meier curve(s) was/were given; (2) the sample size was given; (3) the patients had severe disease or were operative candidates who did not get surgery. When several articles were reported for the same group of patients, only the most recent article was used. The survival curves from the selected series were duplicated by digitizing the original curves in the article. Then, an average curve was computed, weighted by sample sizes, and the mean survival time was calculated by measuring the area under this average curve. For each individual patient, a lifetime was assigned by randomly sampling the survival time on the weighted average survival curves based on the probability of survival. QALYs for patients with unoperated heart valve disease were linearly interpolated between preoperative NYHA class and class IV. If preoperative NYHA class was not

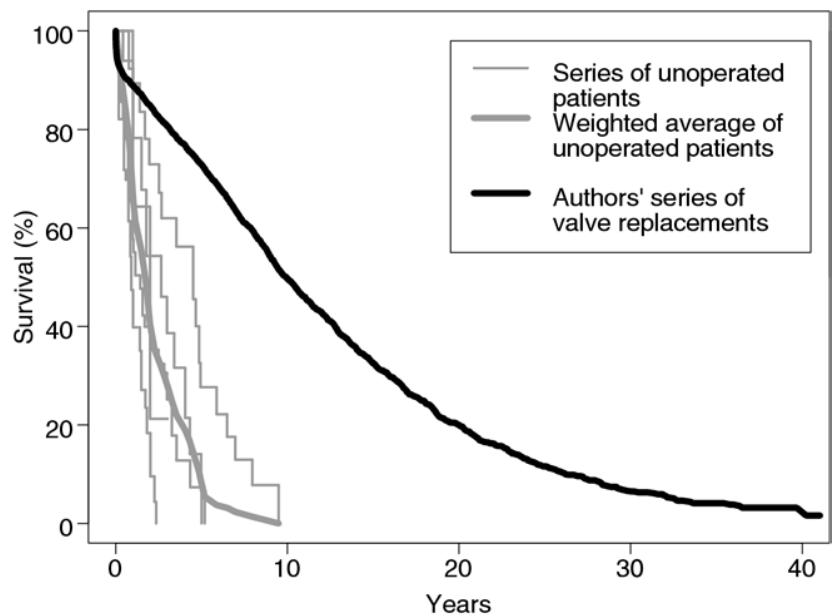

Figure 3. Survival of patients with severe, unoperated aortic valve disease from the literature ${ }^{7-9}$ (the thin light gray curves are series from literature, and the thick light gray curve is the weighted average) and survival of 4617 patients after AVR from the authors' series (thick black curve).

available, it was randomly sampled on the basis of the probability of known preoperative NYHA class.

The simulation was repeated 100 times. The numbers in the "Results" section were based on 1 simulation, and the 95\% ranges were added to the CERs. The study was approved by the institutional review board.

\section{Results}

Survival (mean \pm standard error) after AVR was $50 \% \pm$ $0.9 \%, 20 \% \pm 1.0 \%, 6.8 \% \pm 0.8 \%$, and $3.9 \% \pm 0.8 \%$ at 10 , 20, 30, and 40 years, respectively (Figure 3 ). For the 2382 patients who died, a total of 17,525 follow-up years were observed. For the 2235 censored patients, a total of 14,146 follow-up years were documented, and an additional 21,652 (95\% range was 21,090-22,401) years were imputed using the Gompertz regression model. The mean survival years were 12.4 (9.4 QALYs) for AVR, and the majority of the lifetimes were in NYHA classes I and II (Figure $4, A$ ).

The literature search found 126 studies on unoperated aortic heart valve disease that were published between 1951 and 2004. Five series of aortic stenosis ${ }^{7-9}$ that matched the inclusion and exclusion criteria were used to compose the weighted average curve (Figure 3), and 2 studies for aortic regurgitation were not included because of the small sample size and short follow-up times. The mean survival time was 2.2 years (1.2 QALYs) for aortic valve disease, and the majority of the life years were in NYHA class IV (Figure $4, B$ ).

The total life years after AVR were 53,323, which was equivalent to 39,505 QALYs (total area of the dark gray bars in Figure 5, A). The total expected life years without surgery were 10,157 , which was equivalent to 6159 QALYs 

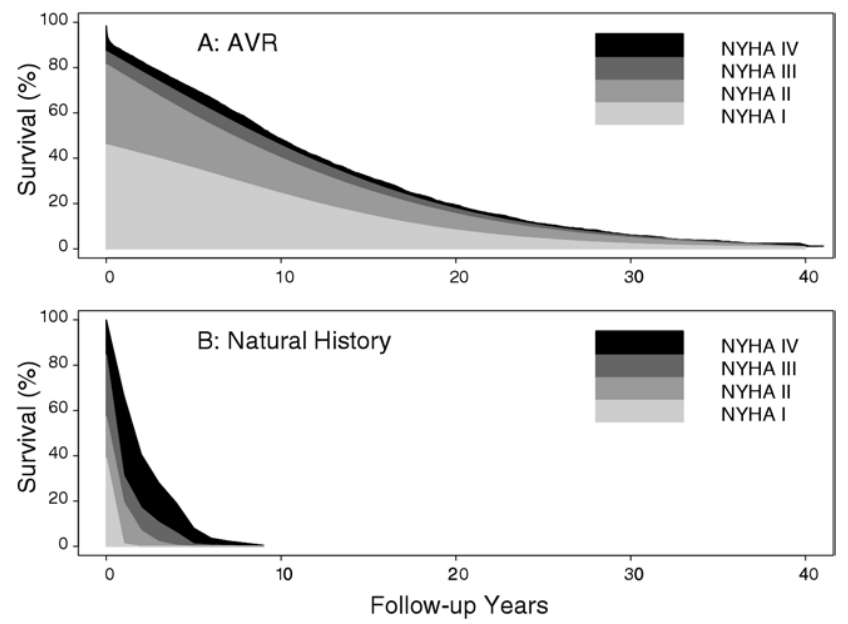

Figure 4. A, Observed survival after AVR (black curve in Figure 3) by follow-up NYHA class. The areas of the different shadings are proportional to the patients' NYHA status during the follow-up years. For example, the majority of patients lived with NYHA class I or II during their remaining lifetime (big areas of light gray colors). B, Estimated survival (thick gray line in Figure 3) by follow-up NYHA class as if patients in (A) did not undergo operation. The majority of patients would have lived with NYHA class IV during their remaining lifetime (big area of black color). AVR, Aortic valve replacement; NYHA, New York Heart Association.

(area of the dark gray bars in Figure 5, B). The total QALYs gained by heart valve replacement was the difference, or 33,346 QALYs, shown by the patient's age at surgery inltl Figure 6, $A$. The average QALYs gained (Figure 6, $B$ ) was computed by dividing the bars in Figure $6, A$ by those in Figure 2, the number of patients at each surgical age.

The total lifetime cost of AVR was \$451 million in 2005 dollars (Figure 7, A): \$277 million for the original surgery; $\$ 50$ million for ongoing maintenance; and \$124 million for treating valve-related events. The average cost per life year (Figure 7, B) is computed by dividing the bars in Figure 7, $A$ by those in Figure 2. The CER by age at surgery was calculated by dividing the lifetime costs at each age group (Figure $7, A$ ) by the lifetime QALYs gained at that age group (Figure 6, A), and is shown in Figure 8. The overall CER was $\$ 13,528$ per QALY and increased with age, up to $\$ 19,826$ per QALY for octogenarians and $\$ 27,182$ per QALY for nonagenarians.

\section{Discussion}

Cost-effective analysis has long been used to compare 2 treatments on the basis of their economic and clinical outcomes. The results of a cost-effective analysis are summarized as a CER, of which the numerator is the difference of the costs of the 2 treatments and the denominator is the difference of the QALYs. Thus, the CER provides the cost
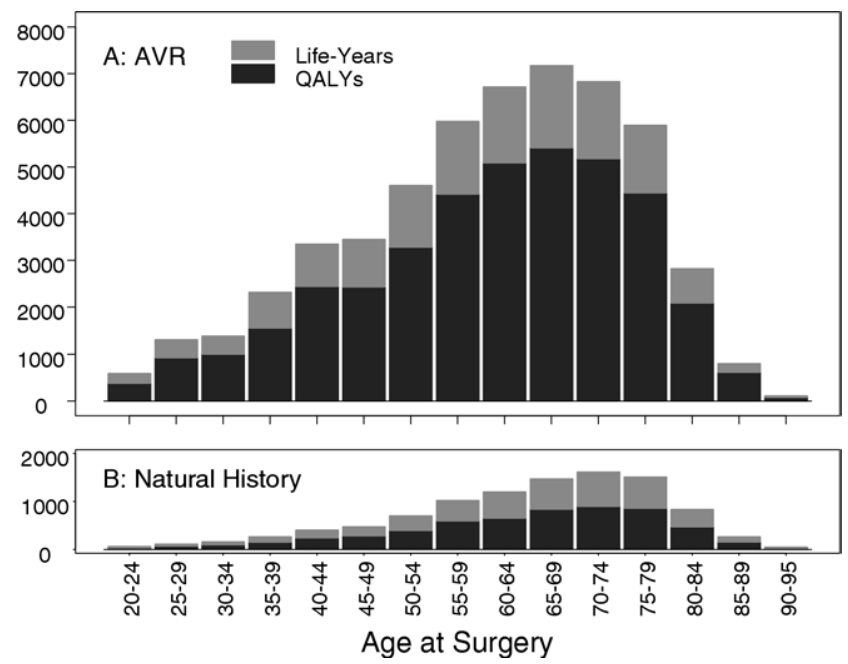

Figure 5. A, Total life years (light gray bars) and respective QALYs (dark gray bars) after AVR by age at surgery. This shows approximately $75 \%$ diminution from perfect health across all age groups after AVR. B, Total life years (light gray bars) and respective OALYs (dark gray bars that are superimposed on the light gray bars) for natural history by age at surgery. This shows approximately $60 \%$ diminution from perfect health across all age groups. AVR, Aortic valve replacement; $Q A L Y$, quality-adjusted life year.
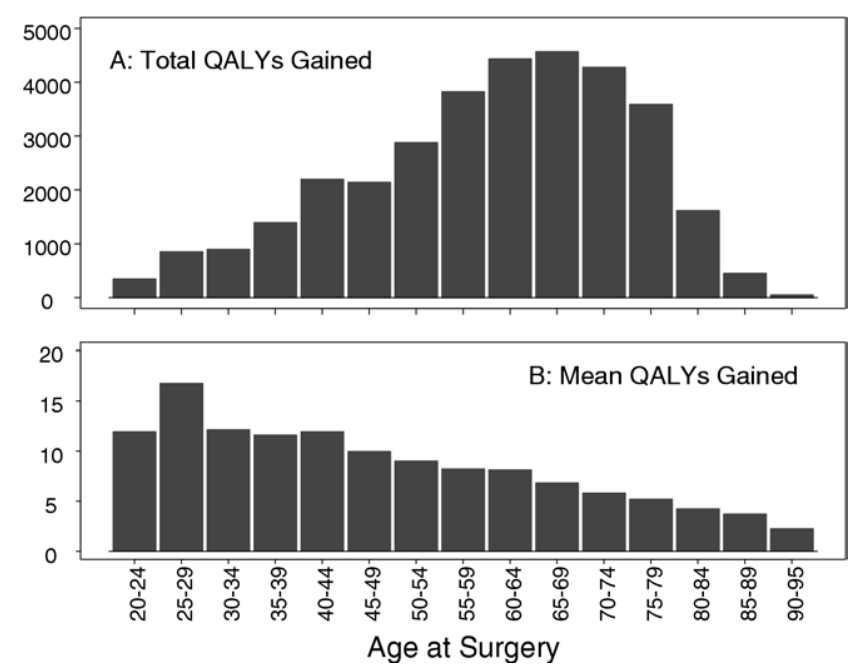

Figure 6. A, Total QALYs gained after AVR by age at surgery. The height of each bar equals the difference of height of the dark gray bars in Figure 5, A and B. B, Mean QALYs gained after AVR by age at surgery (total QALYs gained in Figure 5, A divided by total number of patients in Figure 2 at each age group). AVR, aortic valve replacement; $\boldsymbol{Q A L Y}$, Quality-adjusted life year. 

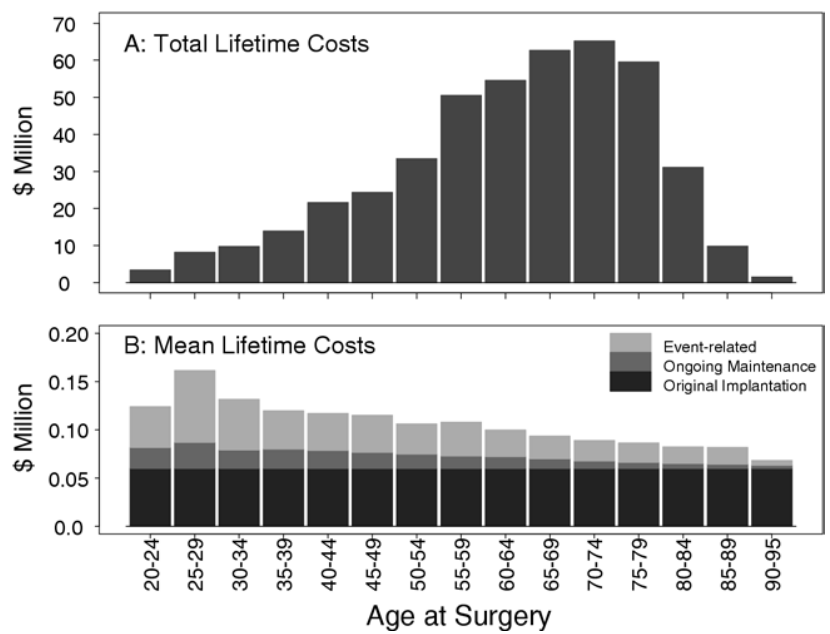

Figure 7. A, Total lifetime costs of AVR by age at surgery. B, Mean lifetime costs of AVR by age at surgery (total cost in Figure 7, A divided by total number of patients in Figure 2 at each age group). The mean cost was further broken down into cost of (1) original implantation; (2) ongoing maintenance (physician visit, echocardiogram, and anticoagulation treatment); and (3) treatment of prosthesis valve-related events (thromboembolism, bleeding, endocarditis, perivalvular leak, valve thrombosis, and reoperation). AVR, aortic valve replacement.

for gaining 1 additional QALY and is commonly used to judge whether a treatment is worth its costs compared with an alternative treatment. For example, the addition of driver's side air bags to safety belts results in net health benefits at an incremental cost of $\$ 24,000$ per QALY saved..$^{10}$ Coronary artery bypass grafting was found to be more costeffective than stenting in patients with multivessel disease. ${ }^{11}$ Established since the early 1960s, AVR has become the standard practice for severe aortic valve disease. This study analyzes the cost-effectiveness of AVR compared with unoperated aortic valve disease. Just as for measuring clinical outcomes, the measurement of economic outcomes in long-term studies faces the challenge of treating censored observations. That is, many patients (sometime the majority) are still alive, and the complete life years are unknown at the time the study is conducted. A statistical model was used to simulate the remaining life years and valve-related complications during the remaining life years. The economic end points were calculated accordingly.

According to our review of the literature and the analysis of our own data, AVR increased the longevity and improved the quality of life of operative candidates. However, the operative and long-term survival are worse in older patients, and advanced age is known to be an independent risk factor for both operative mortality and long-term survival. The primary goal of AVR in very old patients is to improve their quality of life. Thus, the selection of the most elderly for

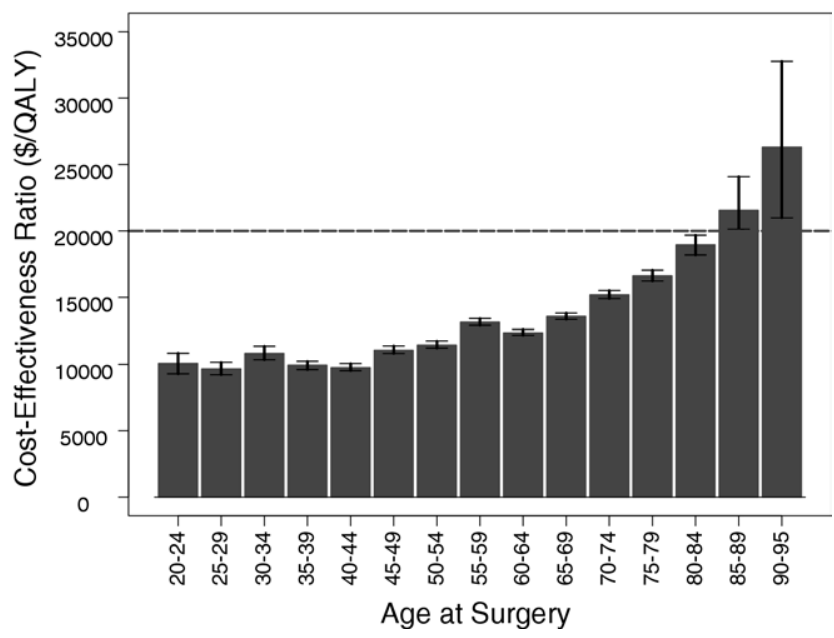

Figure 8. Average CER (cost per QALY) by age at surgery. Error bars indicate $95 \%$ ranges from $100 \times$ simulation. CER less than $\$ 20,000 / 0 A L Y$ was judged as very cost-effective, between $\$ 20,000$ / QALY to $\$ 100,000 / 0 A L Y$ as acceptable, and more than $\$ 100,000$ as not cost-effective. ${ }^{13,14}$ The CERs were less than $\$ 20,000 / 0 A L Y$ for most of the age groups. It was higher for older patients (>80 years), but still less than $\$ 100,000 / 0 A L Y$. CER, Cost-effectiveness ratio; $Q A L Y$, quality-adjusted life year.

AVR is usually conservative considering the balance between risk and benefit. In addition to clinical outcomes, economic end points also affect clinical decision-making, especially in the very elderly. ${ }^{12}$ The current study assessed the life years and QALYs gained after AVR and the costeffectiveness in all ages. Compared with 2.2 years of expected lifetime and 1.2 QALYs with nonsurgical treatment, AVR has extended the mean survival to 12.4 years and 9.4 QALYs. Even for the octogenarians and nonagenarians, the mean survival after AVR was 7.2 years (5.5 QALYs) and 4.8 years (3.9 QALYs), respectively.

The natural history of heart valve disease was derived from older studies, and only a small proportion of the patients in those studies, either surgical candidates or those with advanced disease, were included. Fourteen percent of the study patients had aortic insufficiency, but none of the literature regarding natural history of aortic insufficiency was used because of the small sample sizes and short follow-ups. Because of the small sample sizes and limited information in these studies, the mean survival time is not estimated accurately, and it cannot be adjusted for age, gender, or other factors. Medical treatment also has improved since most of these studies were published, and the life expectancy of patients with severe heart valve disease should be greater than described in the historical literature used for this report. This might lead to an overestimation of cost-effectiveness (lower CER). On the other hand, we counted the lifetime cost of AVR but did not subtract the 
costs of caring for patients with severe, unoperated heart valve disease. This resulted in an underestimation of costeffectiveness (higher CER).

There are several standardized tools widely used to measure quality of life, such as the short form health surveys [36 items and 12 items], EuroQol-5D, Quality of Well-Being Scale, Health Utilities Index, and Health and Activities Limitations Index. The current study included patients from 1961 to 2003, and data for standard quality of life are not available for patients who underwent operation in the earlier days. Thus, the QALYs were estimated by mapping quality of life scores to NYHA functional class. NYHA is not a perfect measure of quality of life, but it has been demonstrated to reflect, or correlate with, quality of life in the literature. $^{4}$

It would be ideal if we could give a precise, personalized estimate of the lifetime cost of AVR. We used the risk model to estimate the lifetime adjusted by patient characteristics; we estimated the number of valve-related complications and the cost of treating those complications. To project the cost during these simulated future life years, valve-related event rates were assumed to occur at the same constant rates as during the observed patient-years. For some valve-related events (eg, structural valvular deterioration), the event rate is not constant and different for mechanical and biological valves. However, constant rates were used for this introductory study. For the cost of hospitalization, we used a flat average number of $\$ 60,000$ for every patient regardless of age, comorbidities, length of stay, and surgical era.

The lifetime cost of AVR decreased with increasing age (Figure 7, B) (older patients have shorter lifetimes to maintain and to experience valve-related complications), but not as fast as the remaining QALYs (Figure 6, B). Thus, the CER increased according to age at surgery (Figure 8). Still, the CER was less than $\$ 20,000$ per QALY for all but the nonagenarians, and it was only $\$ 27,182$ per QALY for them. There are generally accepted thresholds: A treatment costing less than $\$ 20,000 / \mathrm{QALY}$ is considered very costeffective, between $\$ 20,000$ and $\$ 100,000 /$ QALY is acceptable, and more than $\$ 100,000 / \mathrm{QALY}$ is not cost-effective. ${ }^{13,14}$ Despite the limitations, this is well within generally accepted thresholds, making AVR very cost-effective.

This article was our first foray into this new area of econometric research, and we have provided an overview using AVR as the test dataset. Part of the value is to introduce the cardiac surgical community to this type of analysis. Our next step of investigation will be gathering detailed data and refining analysis methodology to provide more specific and practical results for definitive studies.

\section{Conclusions}

AVR was shown to be cost-effective based on generally accepted thresholds for CER, even for the very elderly. However, these thresholds are arbitrary. An econometric method of further quantifying the values of these QALYS, or rather the life years that they are derived from, has evolved and been used to show that these thresholds are actually conservative. ${ }^{15}$

\section{References}

1. Starr A, Edwards ML. Mitral replacement: clinical experience with a ball-valve prosthesis. Ann Surg. 1961;154:726-40.

2. Wu Y, Grunkemeier GL. Statistical analysis of the results of heart valve replacement. Expert Rev Cardiovasc Ther. 2003;1:559-68.

3. Caro JJ, Migliaccio-Walle K, O'Brien JA. The cost of treating heart valve related complications. J Heart Valve Dis. 1996;5:122-7.

4. Maliwa MA, van der Heijden GJ, Bots ML, van Hout BA, Casselman FP, van Swieten H, et al. Quality of life and NYHA class 30 years after mechanical aortic valve replacement. Cardiovasc Surg. 2003;11:381-7.

5. Bonow RO, Carabello B, de Leon AC, Edmunds LH Jr, Fedderly BJ, Freed MD, et al. ACC/AHA guidelines for the management of patients with valvular heart disease: a report of the American College of Cardiology/American Heart Association Task Force on practice guidelines (Committee on management of patients with valvular heart disease). J Am Coll Cardiol. 1998;32:1486-582.

6. Kloster FE, Morris CD. Natural history of valvular heart disease. Circulation. 1982;65:1283-5.

7. Horstkotte D, Loogen F. The natural history of aortic valve stenosis. Eur Heart J. 1988;9:57-64.

8. O'Keefe JH Jr, Vlietstra RE, Bailey KR, Holmes DR Jr. Natural history of candidates for balloon aortic valvuloplasty. Mayo Clin Proc. 1987;62:986-91.

9. Schwarz F, Baumann P, Manthey J, Hoffmann M, Schuler G, Mehmel $\mathrm{HC}$, et al. The effect of aortic valve replacement on survival. Circulation. 1982;66:1105-10.

10. Graham JD, Thompson KM, Goldie SJ, Segui-Gomez M, Weinstein MC. The cost-effectiveness of air bags by seating position. JAMA. 1997;278:1418-25.

11. Yock CA, Boothroyd DB, Owens DK, Garber AM, Hlatky MA. Cost-effectiveness of bypass surgery versus stenting in patients with multivessel coronary artery disease. Am J Med. 2003;115:382-9.

12. Rumsfeld JS. Valve surgery in the elderly: question of quality (of life)? J Am Coll Cardiol. 2003;42:1215-7.

13. Kaplan RM, Bush JW. Health-related quality of life measurement for evaluation research and policy analysis. Health Psychol. 1982;1:61-80.

14. Laupacis A, Feeny D, Detsky AS, Tugwell PX. How attractive does a new technology have to be to warrant adoption and utilization? Tentative guidelines for using clinical and economic evaluations. CMAJ. 1992;146:473-81.

15. Wu Y, Grunkemeier GL, Starr A. The value of aortic heart valve replacement in the elderly: an economic analysis. J Thorac Cardiovasc Surg. 2007;133:603-7. 